\title{
Chiral condensate and spectral flows on $2+1$ flavours Domain Wall QCD from QCDOC
}

\section{RBC and UKQCD Collaborations}

\section{D.J. Antonio, K.C. Bowler, P.A. Boyle, M.A. Clark, B. Joó, A.D. Kennedy, R.D. Kenway, C.M. Maynard, R.J Tweedie}

School of Physics,

The University of Edinburgh

Edinburgh, UK

E-mail:

s0459477@sms.ed.ac.uk

kcbeph.ed.ac.uk

paboyle@ph.ed.ac.uk

mikelph.ed.ac.uk

bjeph.ed.ac.uk

adk@ph.ed.ac.uk

r.d.kenway@ed.ac.uk

cmaynardeph.ed.ac.uk

rjteph.ed.ac.uk

\section{A. Yamaguchi*}

Department of Physics and Astronomy,

The University of Glasgow

Glasgow, UK

a.yamaguchi@physics.gla.ac.uk

\begin{abstract}
We present measurements of the chiral condensate and topological charge history for $2+1$ flavour Domain Wall QCD, and compute spectral flows for the corresponding Hermitian Wilson Dirac operator. We considered several lattice spacings, with the DBW2 and Iwasaki gauge actions and different sea quark masses on a volume of $16^{3} \times 32$ and a fifth dimension of size 8 . These results show the spectral structure on $2+1$ flavours of DWF QCD. We also discuss the dependence of the tunneling rate on lattice spacing and gauge action. All data was generated on the QCDOC machines.
\end{abstract}

XXIIIrd International Symposium on Lattice Field Theory

25-30 July 2005

Trinity College, Dublin, Ireland

* Speaker. 


\begin{tabular}{|l|l|l|l|l|l|} 
Action & $\beta$ & Alg. & $a^{-1} \mathrm{GeV}$ & $m_{\text {ud }} / m_{s}$ & trajs \\
\hline \hline DBW2 & 0.764 & $\mathrm{R}$ & $2.0(2)$ & 1 & 1520 \\
DBW2 & 0.78 & $\mathrm{R}$ & & 1 & 1625 \\
DBW2 & 0.8 & $\mathrm{R}$ & & 1 & 1900 \\
DBW2 & 0.88 & $\mathrm{R}$ & & 1 & 2680 \\
Iwasaki & 2.13 & $\mathrm{R} \_H M C$ & $1.8(1)$ & 1 & 2380 \\
Iwasaki & 2.2 & R_HMC & $2.1(2)$ & 1 & 2960 \\
Iwasaki & 2.3 & R_HMC & & 1 & 1520 \\
\hline DBW2 & 0.764 & R_HMC & $2.0(2)$ & 0.5 & 1800 \\
DBW2 & 0.78 & R_HMC & & 0.5 & 1530 \\
Iwasaki & 2.13 & R_HMC & $1.8(1)$ & 0.5 & 2450 \\
Iwasaki & 2.2 & R_HMC & $2.1(2)$ & 0.5 & 2830
\end{tabular}

Table 1: $N_{f}=3$ flavour datasets on volume $16^{3} \times 32$ with $L_{s}=8$. Inverse lattice spacing estimates $a^{-1}$ are from a unitary extrapolation to the chiral limit.

\section{INTRODUCTION}

Realistic Lattice QCD simulations with dynamical quarks are extremely expensive, and so far it has been common to either use the quenched approximation or use dynamical quark mass values outwith the radius of convergence of chiral perturbation theory. To solve QCD, simulations must be performed with lighter dynamical quark masses, and this requires at least several ten Teraflop-years of computational effort. The QCDOC machine, developed by Columbia University, the University of Edinburgh, RBRC and IBM, enables these lattice simulations with the DWF action on coarse lattice spacings. QCDOC machines of UKQCD started to operate last December and we have ensembles of gauge configurations with $N_{f}=2+1$ dynamical DWF using the DBW2 and Iwasaki gauge actions with various $\beta$ values. These runs were performed on a small lattice volume, $16^{3} \times 32$ with $L_{s}=8$, as a parameter search for large $24^{3} \times 64$ lattice with $L_{s}=16$ or $L_{s}=20$. Other talks in this conference have presented results from this data set $[2,3,5,6]$. In this paper we also discuss spectral flows, topological charge histories, the chiral condensate, and some preliminary results for non-perturbative renormalisation. We discuss some implications for the large volume run.

\section{SIMULATION PARAMETERS}

We use the standard DWF action on a $16^{3} \times 32$ volume with $L_{s}=8$, domain wall height 1.8 , with Pauli-Villars fields, and the standard plaquette plus rectangle gauge action in the special cases of the DBW2 and Iwasaki actions. We used the RHMC algorithm [1] with a trajectory length of 0.5 , and some of the earliest runs used the $\mathrm{R}$ algorithm. A comparison of these algorithms was presented at this conference [2]. The simulation parameters and ensemble sizes are shown in the table 1, and for each entry two ensembles were generated with $a m_{u d}=0.04$ and 0.02 permitting crude unitary chiral extrapolation. The autocorrelation lengths for most observables were in the range 50-100 trajectories [2]. The lattice spacing is set with an error to cover the spread of determinations from $m_{\rho}, f_{\pi}$, and the string tension [3,4]. Measurements for $m_{\text {res }}$ were made with up to four valence quark masses in the range 0.01 to 0.04 on each ensemble $[3,6]$.

\section{RESULTS}

\subsection{Spectral Flows}

We measured the spectral flows of the 4-dimensional hermitian Wilson Dirac operator $H_{w}$ 

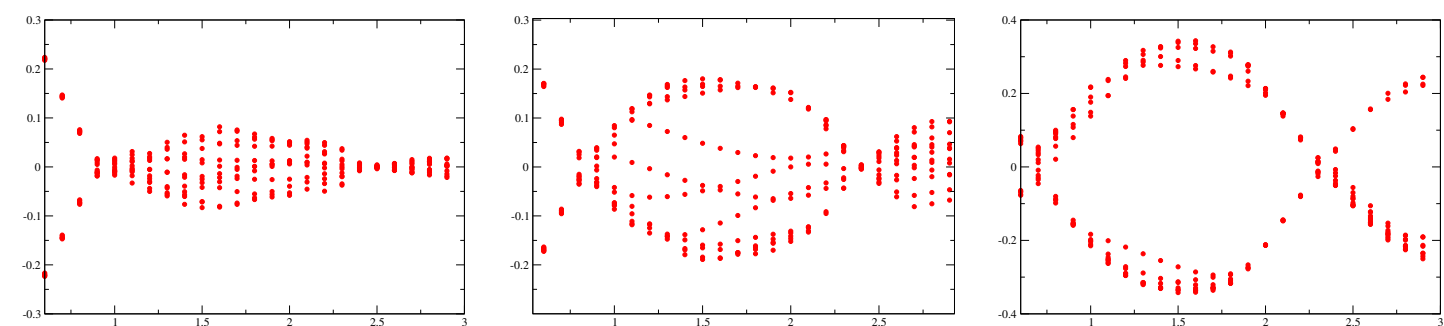

Figure 1: Spectral flow of DBW2. The horizontal axis is the valence domain wall height, $0 \geq M_{5} \geq 3.0$ and the vertical axis is the eigenvalue of $H_{w},-0.3 \geq \lambda_{H w} \geq 0.3$. From left, $\beta=0.72, \beta=0.78$ and $\beta=0.88$.
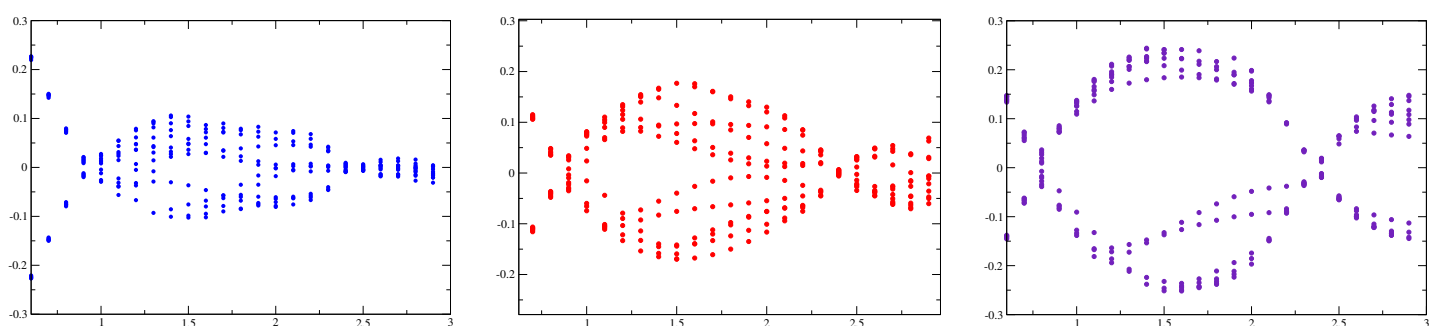

Figure 2: Spectral flow of Iwasaki $\beta=2.13, \beta=2.2$, and $\beta=2.3$. Axes are the same as fig 1 .

using the Chroma code on QCDOC, and saw no significant dependence on the $m_{u d}$ at fixed $\beta$. We display only mass degenerate points in what follows. DBW2 with $\beta=0.72$ show very narrow (to non-existent) gaps in the spectrum. This suggests a high density of near zero modes, such that on typical gauge configurations there is no opening. These near zero modes of the transfer matrix in the fifth dimension cause large $m_{\text {res }}$ [3]. The simulations with the Iwasaki gauge action and with DBW2 at weaker coupling display open spectral flows, and have correspondingly smaller $m_{\text {res }}$.

We note that a level crossing of the spectral flow represents a configuration with indeterminate topological index for the Neuberger Dirac operator, since different choices of $M_{5}$ will change the sign function entering the action. If such crossings are typical, we infer that this suggests frequent topological change. The spectral flows from DBW2 with $\beta \geq 0.8$ suggest a very low topological tunnelling rate and we will later show the topological charge history. This creates a trade-off between chiral symmetry and topological tunneling, and might require compromise.

Comparison between DBW2 fig 1 with Iwasaki fig 2, at nearly matched lattice spacing show that with Iwasaki the opening is slightly narrower than with DBW2. This is consistent with the slightly larger $m_{\text {res }}$ for Iwasaki than DBW2. $m_{\text {res }}$ is so sensitive to $a^{-1}$ that the gain in $m_{\text {res }}$ from from using DBW2 over Iwasaki can be recovered with a small change in lattice spacing. Fig. 1 for $\beta=0.88$ has wide opening and $m_{\text {res }}=1.4 \cdot 10^{-3}$, while $\beta=0.72$ has $m_{\text {res }}=1.1 \cdot 10^{-2}$.

We see more frequent level crossings for Iwasaki configurations than DBW2, especially at finer lattice spacing, and this suggests more topological activity is expected for Iwasaki evolution than DBW2 at finer lattice spacings. We measure the topological charge in the following section.

\subsection{Topological Charge History}

We computed the topological charge as defined by the $O\left(a^{2}\right)$ improved topological charge density, and histories for DBW2 $\beta<0.88$ are shown in fig. 3, displaying reasonable topological activity. However, with DBW2 $\beta=0.88$ there is only one topological event within 3000 trajectories, see fig. 3, proving that the algorithm was not ergodic in our simulation for reasonable numbers of trajectories. With Iwasaki at $\beta=2.3$, fig 4 , which is finer than DBW2 at $\beta=0.88$, there is more frequent topological change. This means that DBW2 action freezes its topology more rapidly than 

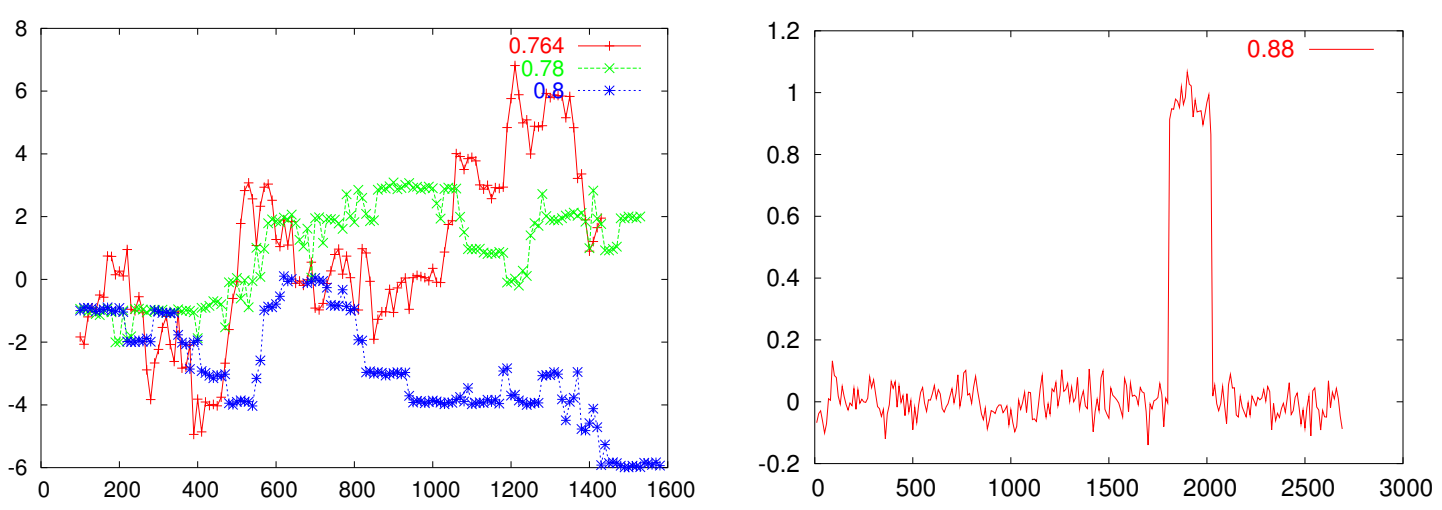

Figure 3: LEFT:Topological charge history for DBW2 with $\beta<0.88$ with $m_{u d}=m_{s}=0.04$. RIGHT: DBW2 with $\beta=0.88$ and $m_{u d}=m_{s}=0.04$.
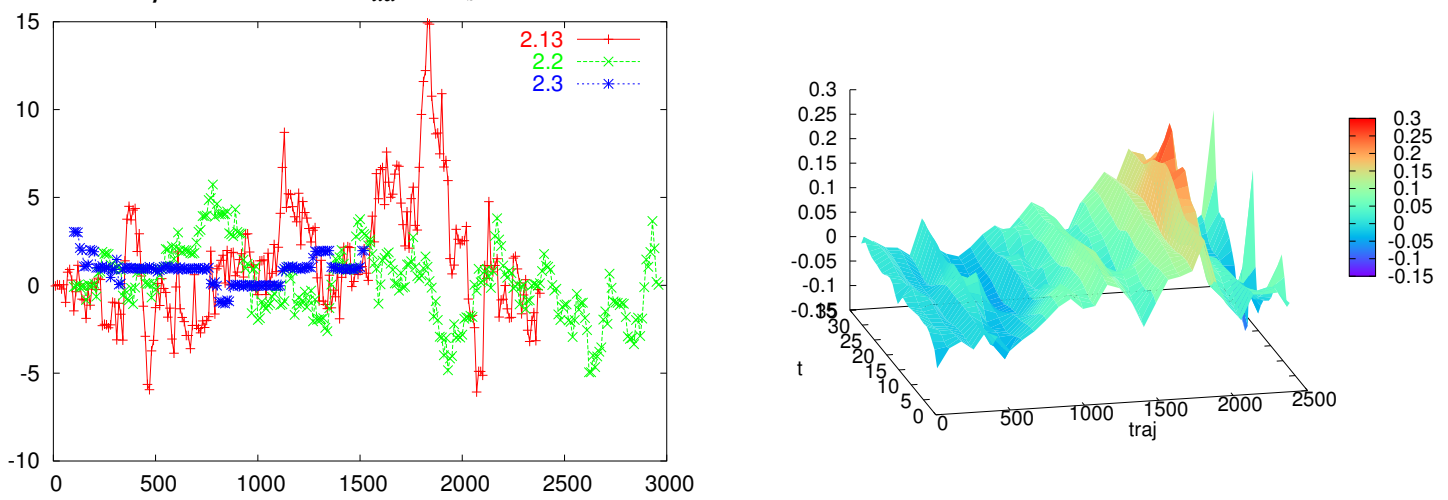

Figure 4: LEFT: Topological charge histories for Iwasaki $\beta=2.13,2.2$, and 2.3 with $m_{u d}=m_{s}=0.04$. RIGHT: Timesliced topological charge density for Iwasaki $\beta=2.13, m_{u d}=m_{s}=0.04$.

the Iwasaki gauge action, while at coarser lattice spacings the gain in residual mass from DBW2 is marginal, leading to one of the main conclusions of our parameter search: to use Iwasaki.

We also measured the histories of the timesliced spatial integral of the topological charge density with similar conclusions, see fig. 4 .

\subsection{Chiral Condensate $\langle\bar{\psi} \psi\rangle$}

The behaviour of the chiral condensate $\langle\bar{\psi} \psi\rangle$ is an important probe to ensure that spontaneous and residual chiral symmetry breaking can be correctly disentangled. We measure $\langle\bar{\psi} \psi\rangle$ as a function of both valence $L_{s}$ and valence $m_{f}$ parameters. Examples of the $m_{f}$ dependence of $\bar{\psi} \psi$ for a number of values for $L_{S}$, and the approach of $\bar{\psi} \psi$ to its asymptotic value in $L_{S}$ are shown (fig 5). A $25 \%$ correction is seen in $\bar{\psi} \psi$ at $L_{s}=8$, though this would be mostly absorbed by the shift in $m_{\text {res }}$. This correction is drastically reduced for $L_{s} \geq 16$. We compare the directly measured $\langle\bar{\psi} \psi\rangle$ at $L_{s}=8$ with the Gell-Mann-Oakes-Renner relation in tab. 2,

$$
f_{\pi}^{2} \frac{m_{\pi}^{2}}{48\left(m_{f}+m_{r e s}\right)}=\langle\bar{\psi} \psi\rangle .
$$

We find that the GMOR is violated at $L_{s}=8$, but we expect that with increasing $L_{s}$ the data may become more consistent with the GMOR as the residual chiral symmetry breaking becomes small compared with the condensate. This is the subject of further study.

Our preliminary findings are that the condensate displays sizable residual chiral symmetry breaking for $L_{s}=8$. The violations of Gell-Mann-Oakes-Renner relation suggests $L_{s}=8$ may 

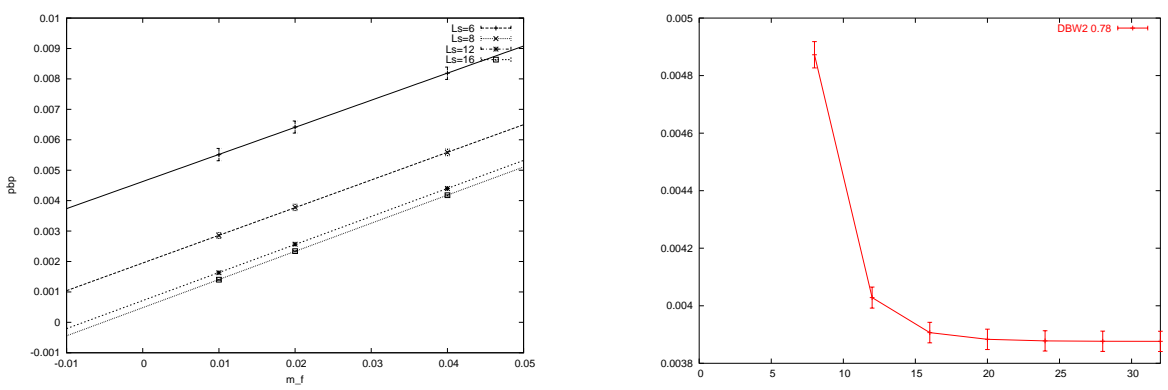

Figure 5: LEFT: $\langle\bar{\psi} \psi\rangle$ for Iwasaki with $\beta=2.13$ and dynamical $m_{u d}=m_{s}=0.04$ with $L_{s}=8$ as a function of valence $m_{f}$ and $L_{s}$. At $L_{s}=8$ residual chiral symmetry breaking in the condensate are somewhat larger than one expects based on the shift in $m_{\text {res }}$ RIGHT: $\langle\bar{\psi} \psi\rangle$ for DBW2 with $\beta=0.78$ and $m_{u d}=m_{s}=0.04$ with $L_{s}=8$ as a function of valence $L_{s}$. Residual chiral symmetry breaking becomes small compared with with the condensate for $L_{s} \geq 16$, and the effect is consistent with an exponential fall off.

$\begin{array}{llll}\text { Action } & \beta & \frac{b}{48} f_{\pi}^{2} & \langle\bar{\psi} \psi\rangle L_{s}=8 \\ \text { DBW2 } & 0.764 & 4.6(4) \cdot 10^{-4} & 1.097(6) \cdot 10^{-3} \\ \text { Iwasaki } & 2.13 & 4.5(8) \cdot 10^{-4} & 1.09(2) \cdot 10^{-3} \\ \text { Iwasaki } & 2.2 & 3.1(4) \cdot 10^{-4} & 1.00(2) \cdot 10^{-3}\end{array}$

Table 2: The Gell-Mann-Oakes-Renner relation

be too short for physics sensitive to the chiral condensate, but we expect that for $L_{s}=16$ and above condensate physics will be well behaved. Our production run that follows on from this parameter search uses the Iwasaki gauge action and $L_{s}=16$ addressing both the topology and chiral condensate concerns.

\subsection{Nonperturbative Renormalisation}

Nonperturbative renormalisation has been used in previous DWF calculations $[9,10]$ to greatly improve predictions of standard model parameters. We calculated preliminary values for $Z_{q} / Z_{A}, Z_{q} / Z_{S}, Z_{q} Z_{m}$ and $Z_{q} / Z_{P}$. A theory with chiral symmetry has the relation,

$$
Z_{S}=Z_{P}, Z_{m}=1 / Z_{S}
$$

After removing the pole term coming from $\langle\bar{\psi} \psi\rangle$ and pion contributions, we can see the relation eq 3.2 between $Z_{S}$ and $Z_{P}$ is held on these lattices, fig 6 . The signal $Z_{q}$ and $Z_{m}$ was very noisy in quenched calculations and $N_{f}=2$ calculation, so we avoid analysing $Z_{q}$ and $Z_{m}$ directly, instead using $Z_{A}$ from the Ward identity [3], $Z_{S} / Z_{A}, Z_{P} / Z_{A}$ and $Z_{m} Z_{A}$ to get $Z_{S}, Z_{P}$ and $Z_{m}$.

\section{Conclusion}

These small lattice simulations with $N_{f}=2+1$ dynamical DWF were generated to search parameter space for a larger production run. With $L_{s}=8$ the residual mass $m_{\text {res }}$ is uncomfortably large and $\langle\bar{\psi} \psi\rangle$ displays residual chiral symmetry breaking effects. In this paper we have presented spectral flows, topological charge history, $\langle\bar{\psi} \psi\rangle$ and NPR, and these have had a direct bearing in the parameter choice made for large simulations currently under way. The parameter search is successfully complete, and has been exploited for a number of useful publications in this conference $[3,7,5,6,4,8]$. Simulations with better chiral symmetry will be obtained on our $2+1$ flavor simulations on $24^{3} \times 64$ with $L_{s}=16$ and the Iwasaki gauge action.

Such calculations would not be possible without both our cost effective and scalable QCDOC, and the efficiency of RHMC. While obtaining a well controlled continuum limit with a chiral 

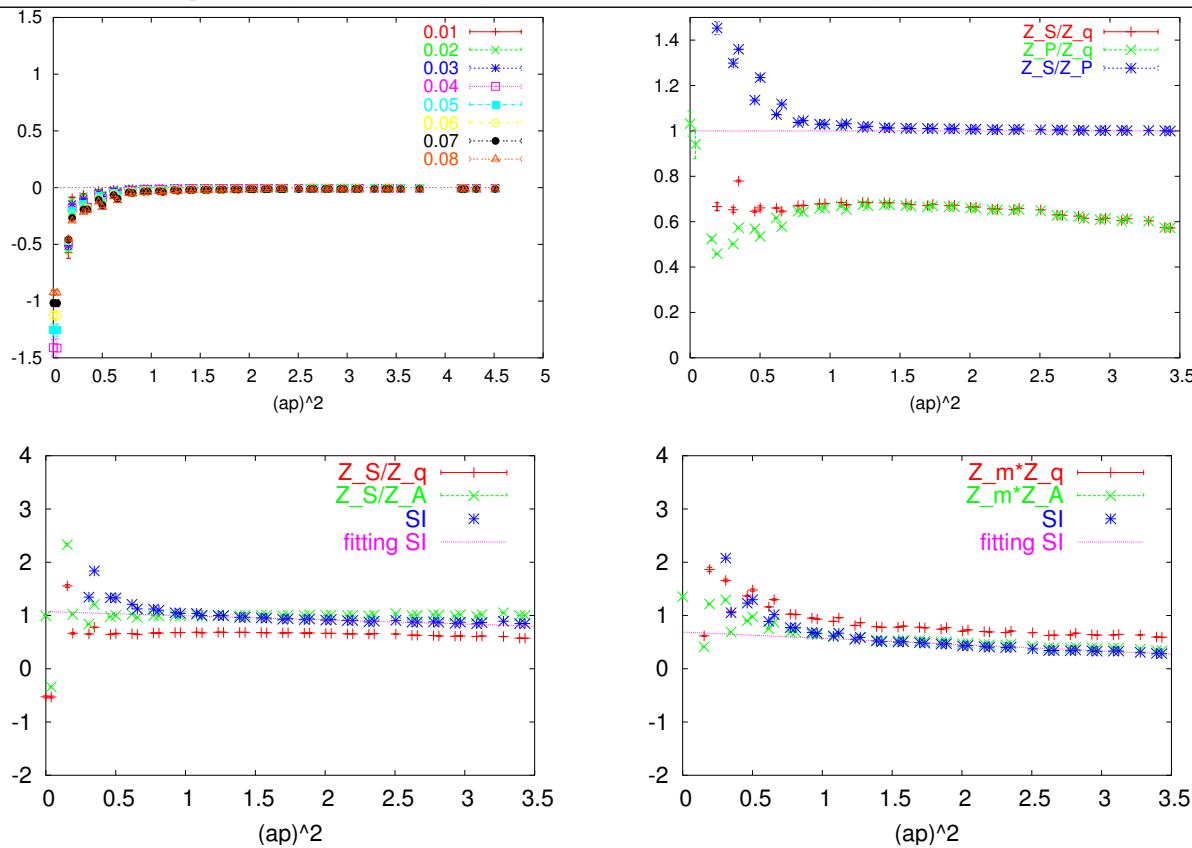

Figure 6: Iwasaki $\beta=2.2, m_{u d} / m_{s}=1,0.01 \leq m_{v a l} \leq 0.08$ : TOP,LEFT: $\Lambda_{A}-\Lambda_{V}$, : TOP,RIGHT: $Z_{S} / Z_{P}$, : BOTTOM,LEFT: $Z_{S} / Z_{A}$, the raw data, the renormalisation group independent (scale independent) improvement, and its fitting. : BOTTOM,RIGHT: $Z_{m} Z_{A}$, the raw data, the renormalisation group independent (scale independent) improvement, and its fitting.

formulation (either using DWF or overlap) will certainly require that in future even larger machines be developed, nevertheless there will be exciting physics produced with QCDOC.

\section{ACKNOWLEDGMENTS}

We thank Sam Li and Meifeng Lin for help generating the datasets used in this work. We thank Dong Chen, Norman Christ, Saul Cohen, Calin Cristian, Zhihua Dong, Alan Gara, Andrew Jackson, Chulwoo Jung, Changhoan Kim, Ludmila Levkova, Xiaodong Liao, Guofeng Liu, Robert Mawhinney, Shigemi Ohta, Konstantin Petrov and Tilo Wettig for developing with us the QCDOC machine and its software. This development and the resulting computer equipment used in this calculation were funded by the U.S.DOE grant DE-FG02-92ER40699, PPARC JIF grant PPA/J/S/1998/00756 and by RIKEN. This work was supported by PPARC grant PP/G/O/2002/00465.

\section{References}

[1] M. A. Clark, A. D. Kennedy and Z. Sroczynski, hep-lat/0409133.

[2] M. A. Clark et al, these proceedings.

[3] Presentation by R. Tweedie, these proceedings.

[4] Presentation by K. Hashimoto, these proceedings.

[5] Presentation by C. Maynard, these proceedings.

[6] Presentation by P. Boyle, these proceedings.

[7] Presentation by M. Lin, these proceedings.

[8] Presentation by S. Cohen, these proceedings.

[9] T.Blum, N. Christ, C. Dawson, et al; Phys.Rev. D66 (2002) 014504

[10] T.Blum, N. Christ, C. Dawson, et al; hep-lat/0411006. 